\title{
Preface to JAISE 13(1)
}

\author{
Vincent Tam ${ }^{\mathrm{a}}$, Hamid Aghajan ${ }^{\mathrm{b}}$ and Juan Carlos Augusto ${ }^{\mathrm{c}}$ \\ a Department of Electrical and Electronic Engineering, Faculty of Engineering, the University of Hong Kong, \\ China \\ b imec, IPI, Department of Telecommunications and Information Processing, Gent University, Belgium \\ ${ }^{c}$ Department of Computer Science and Research Group on Development of Intelligent Environments, Middlesex \\ University, $U K$
}

\section{This Thematic Issue}

The latest advances and developments in data analytics and management, location-aware computing services, networking, recommender systems, social networking, etc. have fuelled the unprecedented growth of various interesting mobile and web applications for location-aware computing and mobile services recommendation in different service domains. On the other hand, the development of such attractive and valuable applications through the mobile and/or web platform will surely arise many challenges to both the theory and practice of location-aware computing and mobile services recommendation technologies while stimulating meaningful discussions among practitioners and researchers across different disciplines in ambient intelligence, IoT and smart environments. In many real-world location-aware applications or recommendation systems, it is always crucial to carefully consider the various criteria, filters or models for analysing the complex relationships among essential data attributes such as the spatial and temporal information of a moving subject before providing any appropriate recommendation/services in the underlying application. Tourism and user trust recommendations are two promising application domains in which careful selections of criteria or data models can lead to offering valuable services to potential customers in smart cities. In addition, the appropriate uses of data models or filters in the underlying location-aware applications can provide optimum performance in road networks or other smart environments.
This Thematic Issue presents some of the latest advances and challenges in the application of smart environments and ambient intelligence to locationaware computing technologies and mobile services recommendation. We wish to thank our colleagues Andrés Muñoz (Catholic University of Murcia, Spain), Honghao Gao (School of Computer Engineering and Science, Shanghai University, China), Wenbing Zhao (College of Engineering, Cleveland State University, USA), and Yuyu Yin (School of Computer Science, Hangzhou Dianzi University, China) for their service in managing this Thematic Issue as guest editors.

\section{Upcoming issues}

The following is a list of upcoming issues of JAISE:

- March 2021: Regular Issue.

- May 2021: Thematic Issue on Trustworthy Computing for Secure Smart Cities.

- July 2021: Regular Issue.

- September 2021: Thematic Issue on Deep Learning-based Real-time Visual Analytics in a Smart City.

- November 2021: Regular Issue.

More information on the call for papers to the future thematic issues is available on the webpage of JAISE at: http://www.iospress.nl/journal/journal-of-ambientintelligence-and-smart-environments/. 